\title{
Analysis of the instrumentation used in randomized controlled trials to assess pain in the osteopathic intervention: A short review
}

\author{
Rui José Santiago ${ }^{1}$, António Torres Marques², J. Santos Baptista ${ }^{3}$, J. Torres Costa ${ }^{4}$
}

\begin{abstract}
${ }^{1}$ Associated Laboratory for Energy, Transports and Aeronautics (PROA/LAETA), Faculty of Engineering, University of Porto, PT (rui.santiago@gmail.com) ORCID 0000-0002-8227-5707, 2Associated Laboratory for Energy, Transports and Aeronautics (PROA/LAETA), Faculty of Engineering, University of Porto, PT (marques@fe.up.pt), ${ }^{3}$ Associated Laboratory for Energy, Transports and Aeronautics (PROA/LAETA), Faculty of Engineering, University of Porto, PT (jsbap@fe.up.pt) ORCID 0000-0002-8524-5503, ${ }^{4}$ Faculty of Medicine, University of Porto, Porto, PT (zecatoco@sapo.pt)

https://doi.org/10.24840/978-972-752-260-6 0001-0004
\end{abstract}

\begin{abstract}
Introduction: Osteopathy is an emergent health-care profession, present in most of the developed world and since 2013, regulated in Portugal. Osteopaths intervene in a range of health issues in which pain is a very common aspect. Pain is a worldwide problem, affecting all aspects of society. Measurement of pain objectively is yet not possible; even considered unreliable in many circumstances, the standard is selfreported questionnaires. A variety of different scales were used to measure the intensity of pain in Osteopathic research; however, the criteria of the options are not always clear or reported. This review aims to analyze and critically compare the different characteristics of the most used questionnaires by osteopathic researchers for assessing the intensity of pain in randomized controlled trials (RCT). Methodology: A literature search was conducted using 7 electronic databases. This search was conducted for RCT articles studying the efficacy/effectiveness of the osteopathic manipulative treatment (OMT) intervention in pain. The findings followed the PRISMA statement. Included studies were assessed for the risk of bias (RoB) using the Jadad score. Results and Discussion: 123 studies were identified, and after removal of duplicates and application of the eligibility criteria, 21 articles were included for this review. Nine studies used the Visual Analogue Scale (VAS) $10 \mathrm{~cm}$ scale, seven the Numeric Rating Scale (NRS) 11 points scale, the remaining used other options. Although similar, there are differences in these two scales that may affect the outcomes. Justification of the choice of the evaluation instrument was not always present and not associated with the methodology and the target population. The overall quality of the studies, in terms of RoB, was considered good. Conclusion: VAS and NRS are the choices of most authors. Authors in Osteopathy, or other health care professions should be very clear about the reasons behind the choices for measuring the intensity of pain; these should fit the objectives and study design.
\end{abstract}

Keywords: Osteopathic intervention, Pain scales, Assessment of pain.

\section{INTRODUCTION}

Osteopathic medicine is a healthcare form centered in the patient rather than on the disease. The professional of Osteopathy intervenes through a range of manual techniques within the Osteopathic Manipulative Treatment (OMT) to support homeostasis and improve physiologic function, as well as advise in lifestyle, diet, counseling, posture and physical activity (WHO, 2010). Osteopathic medicine is regulated in more than 15 countries, including the United States, Canada, Australia, New Zealand, and 8 European countries, among them is Portugal, since 2013, it is also being taught in higher education in Portugal, since 2016 (OIA, 2013; FORE, 2018). Still, this world-wide emergent primary care health profession needs more evidence-based support for its claims and secure its place within the health care professions. Due to the myriad of contexts necessary to study to have a more informed decision if Osteopathic Manipulative Treatment (OMT) is the best clinical option, more studies should be designed and implemented to understand its effectiveness and or efficacy in general symptoms and pain particularly. Every year, millions suffer from acute or chronic pain, having a considerable impact on costs and budgets for any country as it affects work productivity and demands resources for health care, prevention, and rehabilitation. In the USA alone, chronic pain affects 100 million people, and it costs society at least $\$ 560-\$ 630$ billion, every year (AAPM, 2018). Attempts to understand and define pain are one of the oldest challenges of medicine and other subjects once it plays a crucial role in culture and identity (Raffaeli and Arnaudo, 2017). The measurement of pain in clinical research of musculoskeletal disorders (MSD's) has been a central question that many medical specialities tried to explore. Self-reporting assessment of pain is still the 'gold-standard' but, 
according to Cowen et al., (2015), to process external information and report such personal experience may be unreliable in many circumstances. The objective of this review is to analyze the different tools used in the last five years to assess pain in studies analyzing the efficacy or effectiveness of the osteopathic intervention. For this work, pain is not subject to location, pathology, or another contextual variable. To the author's knowledge, this is the first critical systematic review to appraise the use of these measuring systems used to quantify the intensity of pain in Osteopathic interventions in Randomized Controlled Trials (RCT).

\section{METHODOLOGY}

Literature searches were performed from the $3^{\text {rd }}$ to the $10^{\text {th }}$ of March of 2018 to identify all randomized controlled trials (RCT's) where the pain was one of the outcomes of the analysis of efficacy or effectiveness for the OMT intervention. The research methods and reporting of this study followed the PRISMA guidelines (Liberati et al., 2009).

\section{Search Strategy}

Twenty-one Relevant studies were identified through a comprehensive bibliographic search on the following electronic databases: SCOPUS, Cochrane Central Register of Controlled Trials, MEDLINE, PEDro, OSTMED.DR, Science Direct and Web of Science. The terms searched were osteopath*, Randomized Controlled Trial OR RCT, Pain, Efficacy OR Effect*. The combination of terms used was adapted to the different database search engine specifications. Duplicated records from the searches were identified and removed. The authors considered that searching for the last five years was appropriate for the objectives of this work.

\section{Eligibility criteria}

The search limits applied after the removal of duplicates were: (1) Published before 2012; (2) Not published in English; (3) Full paper not accessible; (4) Participants not human and (5) Subject out of theme. The Eligibility criteria applied was: (1) The study must include the application of OMT, not only a single technique; (2) Randomized and Controlled Trials study design exclusively and must assess for efficacy or effectiveness of the OMT intervention; (3) Study outcomes must include pain and use subjective or objective measurements; (4) Study must be approved by an Ethics Committee or state the use of written consent.

\section{Risk of Bias}

The included trials were assessed for their individual Risk of Bias (RoB) using the five-point Jadad score (Jadad et al., 1996).

\section{Data Synthesis}

Findings of this review were summarised in narrative form, and data reported as means and percentages.

\section{RESULTS}

\section{Study Selection}

The search strategy for the current review identified 527 articles, all from database searching. Selection based on relevance to the topic and the exclusion of duplicates left 123 articles. After 
reading the full text and applying the inclusion criteria to all selected publications, 21 articles were included in this analysis.

\section{Pain Assessment}

All the assessment methods of pain in the RCTs included in this article were all in the format of questionnaires and therefore, subjective. The results of this study show that there is a predominance of the use of different models of the VAS and the NRS scores, as shown in Figure 1. Most authors did not present a justification for their choice of scale.

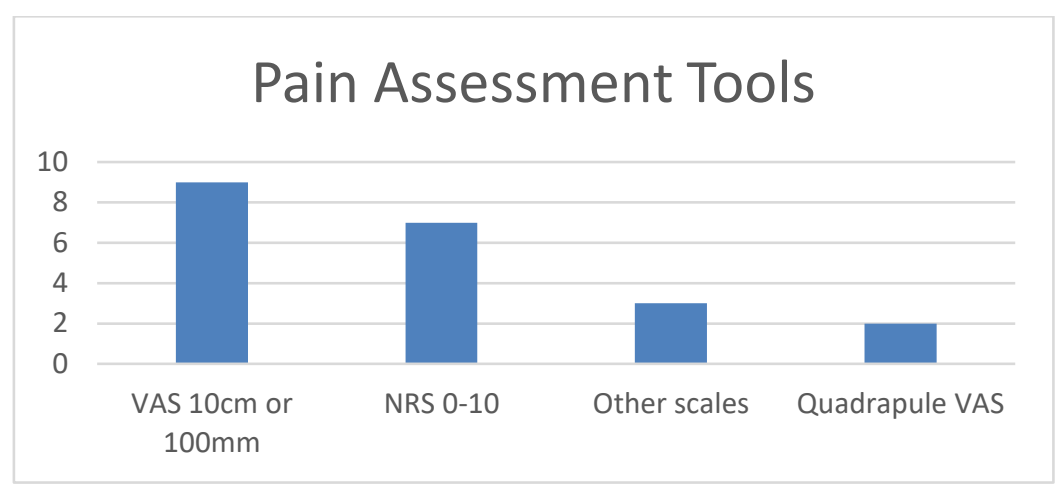

Figure 1. Represents the incidence of use of each of the pain assessment tools in the included studies.

\section{DISCUSSION}

\section{Subjective measurement of pain}

At least 30 different instruments have been used for the past 30 years in the attempt to measure pain. The most commonly used have been VAS, the NRS and Verbal Radical Scale (VRS) but translating these results into useful patient information is more of an art than a science (Zanoli et al., 2002). Hjermstad et al., (2011), concluded that VAS, NRS-11, and VRS-7 all work well for pain intensity. According to this author, the relevant choice is not the type of scale, but the conditions associated, the standardization of anchor descriptors, methods of administrations, time frames, interpretation of cut-offs and clinical significance. In a more recent review of the pain scales in adults, by Karcioglu et al., (2018), the same three questionnaires were compared, and all were reported as valid and reliable. VAS was considered more difficult than the others. The elderly and those with cognitive impairments, communication problems and minorities have found verbal descriptor or rating scales more practical than others.

\section{Comparison of VAS and NRS}

Hawer et al., (2011) described all the questionnaires used for assessing pain in rheumatologic, adult patients, but these same questionnaires have been widely used for pain in other populations. Among them are, VAS and NRS, the two described as generic, one-dimensional pain questionnaire. These were the two most used questionnaires in the included studies; both are widely used and reliable for pain intensity, not the pain experience. NSR has the advantages of being administered both verbally and in writing, the simplicity of the scoring (no need to take measures) and it is equally reliable in the literate and illiterate participant. 


\section{CONCLUSIONS}

These results show that the Visual Analogue Scale $10 \mathrm{~cm}$ and Numeric Rating Scale 11-point were the options of most authors studying the efficacy or effectiveness of OMT in the intensity pain using RCT methodology. These two questionnaires have a few differences that can potentiate a more accurate use depending on the type of population and the study design. The authors recognize limitations in selection of studies, in the time frame of 5 years, papers and in excluding non-English works. Future research in Osteopathy for the intensity of pain should justify the choice of a survey to meet the study design and the specific aspects of the outcomes towards a better-informed practice.

\section{References}

AAPM. (2018). Facts and Figures on Pain. Retrieved from http://www.painmed.org/patientcenter/facts-on-pain/

Cowen, R., Stasiowska, M. K., Laycock, H., \& Bantel, C. (2015). Assessing pain objectively: the use of physiological markers. Anaesthesia, 70(7), 828-847. doi:10.1111/anae.13018

FORE. (2018). REGULATION OF OSTEOPATHY IN EUROPE. Retrieved from ttps://www.forewards.eu/regulation/

Hjermstad, M. J., Fayers, P. M., Haugen, D. F., Caraceni, A., Hanks, G. W., Loge, J. H., . . European Palliative Care Research, C. (2011). Studies comparing Numerical Rating Scales, Verbal Rating Scales, and Visual Analogue Scales for assessment of pain intensity in adults: a systematic literature review. J Pain Symptom Manage, 41(6), 1073-1093. doi:10.1016/j.jpainsymman.2010.08.016

Jadad, A. R., Moore, R. A., Carroll, D., Jenkinson, C., Reynolds, D. J., Gavaghan, D. J., \& McQuay, H. J. (1996). Assessing the quality of reports of randomized clinical trials: is blinding necessary? Control Clin Trials, 17(1), 1-12.

Karcioglu, O., Topacoglu, H., Dikme, O., \& Dikme, O. (2018). A systematic review of the pain scales in adults: Which to use? Am J Emerg Med, 36(4), 707-714. doi:10.1016/j.ajem.2018.01.008

Liberati, A., Altman, D. G., Tetzlaff, J., Mulrow, C., Gotzsche, P. C., loannidis, J. P., . . Moher, D. (2009). The PRISMA statement for reporting systematic reviews and meta-analyses of studies that evaluate health care interventions: explanation and elaboration. PLoS Med, 6(7), e1000100. doi:10.1371/journal.pmed.1000100

OIA. (2013). History and Current Context of the Osteopathic Profession. Retrieved from http://oialliance.org/resources/oia-status-report/

Raffaeli, W., \& Arnaudo, E. (2017). Pain as a disease: an overview. J Pain Res, 10, 2003-2008. doi:10.2147/JPR.S138864

WHO. (2010). Benchmarks for Training in Traditional/Complementary and Alternative Medicine, Benchmarks for Training in $\quad$ Osteopathy. $\quad$ Retrieved from www.who.int/medicines/areas/traditional/BenchmarksforTraininginOsteopathy.pdf

Zanoli, G., Strömqvist, B., Jönsson, B., Padua, R., \& Romanini, E. (2002). Pain in low-back pain: Problems in measuring outcomes in musculoskeletal disorders. Acta Orthopaedica Scandinavica, 73(sup305), 54-57. doi:10.1080/000164702760379576 